\title{
VECTOR BUNDLES OVER THREE-DIMENSIONAL SPHERICAL SPACE FORMS
}

\author{
ESDRAS TEIXEIRA COSTA, OZIRIDE MANZOLI NETO, AND MAURO SPREAFICO
}

Received 12 March 2006; Accepted 13 March 2006

We consider the problem of enumerating the G-bundles over low-dimensional manifolds (dimension $\leq 3$ ) and in particular vector bundles over the three-dimensional spherical space forms. We give a complete answer to these questions and we give tables for the possible vector bundles over the 3-dimensional spherical space forms.

Copyright (c) 2006 Hindawi Publishing Corporation. All rights reserved.

\section{Introduction}

In this work we consider the class of the compact connected three-dimensional manifolds with positive constant curvature, also known as the three-dimensional spherical space forms. These spaces, or subclasses like generalized quaternions or lens spaces, appear in many different contexts in topology and geometry, and have been completely classified; it is thus natural to ask if we can also count the bundles over them. We answer positively to this question, and give tables in Section 5 to describe all the vector bundles of rank less than 3 over any three-dimensional spherical space form. Besides, in Section 2, we show that, under reasonably wide assumptions on the structure group $G$, $G$-bundles over any low (lower or equal to three)-dimensional manifolds can be counted effectively.

\section{Bundles over low-dimensional manifolds}

Let $G$ be a Lie group and $M^{m}$ a closed manifold of finite dimension $m=1,2$, or 3 . Let $\mathscr{B}(M, G)$ be the set of the equivalence classes of principal $G$ bundles over $M$. Recall that $\mathscr{B}(M, G)=[M, B G]$ and, by dimensional reason and since $\pi_{2}(G)=0$, they coincide with the set $\left[M,(B G)_{m}\right], m=1$ or 2 , where $(B G)_{m}$ is the space appearing at level $m$ in the Postnikov decomposition of $B G$. Thus, when $G$ is connected,

$$
\begin{gathered}
\mathscr{B}\left(M^{1}, G\right)=\{0\}, \\
\mathscr{B}\left(M^{2,3}, G\right)=H^{2}\left(M ; \pi_{1}(G)\right) .
\end{gathered}
$$

Hindawi Publishing Corporation

International Journal of Mathematics and Mathematical Sciences

Volume 2006, Article ID 47574, Pages 1-11

DOI 10.1155/IJMMS/2006/47574 
2 Vector bundles over three-dimensional spherical space forms

When $G$ is not connected we need local coefficients. We can proceed as in [7] and use the Larmore spectral sequence [6]. We introduce the following quite general assumption: we assume that the projection $p_{0}: G \rightarrow G / G_{0}$ to the quotient by the connected component of the identity has a continuous section $s: G / G_{0} \rightarrow G$. If this is the case, then $G=G / G_{0} \ltimes_{\alpha} G_{0}$, for some homomorphism $\alpha: G / G_{0} \rightarrow \operatorname{Aut}\left(G_{0}\right)$, and we can apply [7, Proposition 1].

Proposition 2.1. The classifying space $B G$ is the total space of a $G / G_{0}$-bundle over $B\left(G / G_{0}\right)$ with fibre $B G_{0}$ and projection $B p_{0}: B G \rightarrow B\left(G / G_{0}\right)$. Moreover, the splitting map sinduces a cross-section $B s: B\left(G / G_{0}\right) \rightarrow B G$.

Hence, the relevant Postnikov sections are twisted Eilenberg-Mac Lane spaces, and we obtain the exact sequence of sets

$$
\left[M, B G_{0}\right] \stackrel{\left(B i_{0}\right)_{*}}{\longrightarrow}[M, B G] \stackrel{\left(B p_{0}\right)_{*}}{\longrightarrow}\left[M, B\left(G / G_{0}\right)\right] \longrightarrow 0
$$

When $M$ has dimension 1 , this gives

$$
\left[M^{1}, B G\right]=\operatorname{Hom}\left(\pi_{1}\left(M^{1}\right), \pi_{0}(G)\right) / P \pi_{0}(G)
$$

where the action is by conjugation, namely $(\phi, \alpha)(x) \mapsto \phi^{\alpha}(x)=\alpha^{-1} \phi(x) \alpha$, and $P G=$ $G / Z G$ denotes the quotient by the center. When $M$ has dimension 2 or 3 , we need to compute $\left(B p_{0}\right)_{*}^{-1}\left(B p_{0}\right)_{*}([f])$ for $[f] \in[M, B G]_{0}$. By surjectivity, it is enough to compute $\left(B p_{0}\right)_{*}^{-1}([u])$ for all $[u] \in\left[M, B\left(G / G_{0}\right)\right]_{0}$, and as before we can enumerate the elements $[u] \in\left[M, B\left(G / G_{0}\right)\right]_{0}$ by the correspondent elements $\phi_{u} \in \operatorname{Hom}\left(\pi_{1}(M), \pi_{0}(G)\right)$. We can use the Larmore spectral sequence [6] as in [7] (that has trivial differential in this case). We obtain $\left(B p_{0}\right)_{*}^{-1}\left(u_{\phi}\right)=H^{2}\left(M ; \pi_{1}\left(G, u_{\phi}\right)\right)$, and hence

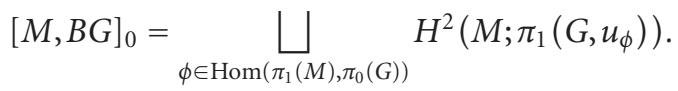

Eventually, we need to get the quotient by the action of $\pi_{0}(G)$ to get free classes. It follows from [6, Theorem 2.2.2] that the operation + commutes with the action of $\pi_{0}(G)$ as follows: $\left(u_{\phi}+b\right)^{\alpha}=u_{\phi}^{\alpha}+1_{*}^{\alpha}(b)$, with $1_{*}^{\alpha} \in \operatorname{Aut}\left(H^{2}\left(M ; \pi_{1}\left(G, u_{\phi}\right)\right)\right)$, and hence the quotient can be taken on the group of the homomorphisms. In summary, we have proved the following theorem.

THEOREM 2.2. Let $G$ be a compact Lie group satisfying the above assumption and $M$ a closed manifold of dimension 1, 2, or 3. Then,

$$
\begin{gathered}
\mathscr{B}\left(M^{1}, G\right)=\operatorname{Hom}\left(\pi_{1}\left(M^{1}\right), \pi_{0}(G)\right) / P \pi_{0}(G), \\
B\left(M^{2,3}, G\right)=\bigsqcup_{\bar{\phi} \in \operatorname{Hom}\left(\pi_{1}(M), \pi_{0}(G)\right) / P \pi_{0}(G)} H^{2}\left(M ; \pi_{1}\left(G, u_{\phi}\right)\right) / \pi_{0}(G) .
\end{gathered}
$$


Notice that the action of $\pi_{0}(G)$ is trivial whenever $\pi_{0}(G)$ is abelian (in particular if $G$ is abelian) and $B G$ is 2-simple.

\section{Twisted cohomology of 3-dimensional spherical space forms}

Let $p: F \rightarrow F / R=G$ be a presentation for a finite group $G$, where $F$ and $R$ are free on the sets $S$ and $T$, respectively. By [3] or [1] we obtain a free resolution of $\mathbb{Z}$ over $\mathbb{Z} G$ as follows. Let $A$ and $B$ denote ordered sets of abstract module's generators, one generator for each element in the corresponding set of the group's generators $S$ and $T$, respectively, let $e$ be a single abstract generator, and define the homomorphisms

$$
\phi_{1}(a)=\left(1-s_{a}\right) e, \quad \phi_{2}(b)=\sum_{a \in A}\left(d_{s_{b}} r_{a}\right) a
$$

where $s_{b}$ and $r_{a}$ denote the elements in the group's generators set corresponding to the abstract basis elements, and we recall that the group derivation is defined on the elements of $F$ by $d_{s} 1=0, d_{s}(u v)=d_{s}(u)+u d_{s} v$, and $d_{s_{i}} s_{j}=\delta_{i j}$, for all $s \in S$. A free resolution of $\mathbb{Z}$ over $G$ is then

$$
\cdots \longrightarrow \mathbb{Z} G[B] \underset{\phi_{2}}{\longrightarrow} \mathbb{Z} G[A] \underset{\phi_{1}}{\longrightarrow} \mathbb{Z} G[e] \underset{\epsilon}{\longrightarrow} \mathbb{Z} \longrightarrow 0 .
$$

Let $\Gamma$ be a finite subgroup of $S_{4}(\mathbb{R})$ operating freely on the three sphere $S^{3}$. The quotient spaces $S_{\Gamma}=S^{3} / \Gamma$ are three-dimensional Riemannian orientable closed manifolds called (orthogonal) spherical space forms [10]. A first complete classification of these manifolds was given implicitly by Hopf [5] and in more details by Seifert and Threlfall [8]. This classification is given by the list of the possible groups $\Gamma$ (see also [4]). They are (for presentations see Section 4)

(1) the cyclic group $C(n)$, the generalized quaternionic group $Q(4 n)$, the binary tetrahedral group $T^{*}(24)$, the binary octahedral group $O^{*}(48)$, and the binary icosahedral group $I^{*}(120)$;

(2) the semidirect products $C(2 n+1) \rtimes C\left(2^{k}\right), k \geq 2, n \geq 1$;

(3) the semidirect products $Q(8) \rtimes C\left(3^{k}\right), k \geq 1$;

(4) the product of any of the above groups with a cyclic group of coprime order.

Since $S_{\Gamma}$ is the three-skeleton of the Eilenberg Mac Lane space $K(1, \Gamma)$, and all the groups appearing in the above list are finite and finitely presented, the $\mathbb{Z} \Gamma$-chain complex for the universal covering space $\widetilde{S}_{\Gamma}\left(\cong S^{3}\right)$ is given by the resolution (3.2). This provides the chain complex only up to level 2 , but this is enough for our purpose since we can dualize the complex to compute the first cohomology groups and eventually apply a generalized version of the Poincaré duality, that holds here without restrictions since the manifolds are orientable, to complete the calculations.

\section{Calculations}

In this section we do the necessary calculations in order to apply Theorem 2.2 for the real vector bundles over the spherical space forms of dimension 3 . Thus, $M=S_{\Gamma}$ ( $\Gamma$ being one 
of the groups listed in Section 3), $G=O_{n}, G_{0}=S O_{n}, G / G_{0}=\pi_{0}(G)=\mathbb{Z} / 2$, and $\pi_{2}(B G)=$ $\pi_{1}(G)=0, \mathbb{Z}$ or $\mathbb{Z} / 2, n=1,2,3$. Notice that $G$ is abelian and $B G$ is 2 -simple in all cases except one, when the action of $\pi_{1}(B G)$ corresponds to a change in the local orientation of the bundle. Actually, this case never arises, as appears from the tables in Section 5.

We proceed with the calculations as follows. Each time, we first compute $\operatorname{Hom}\left(\pi_{1}\left(S_{\Gamma}\right)\right.$, $\left.\pi_{0}(G)\right)=\operatorname{Hom}(A b(\Gamma), \mathbb{Z} / 2)$, that corresponds to the set of the real line bundles over $S_{\Gamma}$. Next, we need the cohomology of $S_{\Gamma}$, twisted by all these line bundles if $n=2$. Let $u$ be an element in $\left[S_{\Gamma}, B\left(G / G_{0}\right)\right]$ that classifies a line bundle, and let $\phi_{u}$ in $\operatorname{Hom}\left(\pi_{1}\left(S_{\Gamma}\right), \pi_{0}(G)\right)$ be the corresponding homomorphism. We need to compute $H^{2}\left(S_{\Gamma} ; \pi_{1}(G, u)\right)$. When $G=O_{2}$, since $(B G)_{1}=G / G_{0}$, the sheaf $\pi_{1}(G, u)$ with fibre $\pi_{1}(G)=\mathbb{Z}$ and group $\pi_{0}(G)=\mathbb{Z} / 2$, acting by the automorphism determined by a representation $\rho: \pi_{0}(G) \rightarrow \operatorname{Aut}\left(\pi_{1}(G)\right)$, corresponds bijectively to (is classified by) the twisting homomorphisms $\phi: \pi_{1}\left(S_{\Gamma}\right) \rightarrow \pi_{0}(G)$, that is, we can identify (in the other cases we just need the trivial sheaf) $H^{2}\left(S_{\Gamma} ; \pi_{1}\left(G, u_{\phi}\right)\right)$ $=H^{2}\left(S_{\Gamma} ; \mathbb{Z}_{\rho_{\phi}}\right)$.

Therefore, for each $\Gamma$ and each allowed representation for it, we give the explicit form of the chain complex described in Section 3, and we compute the twisted homology groups. We will use the following notation for the groups representations: for a subset $W$ of the set of the generators of $\Gamma$, let $\rho_{W}: \Gamma \rightarrow \operatorname{Aut}(\mathbb{Z})$ denote the homomorphism determined by $\rho_{W}(W)=-1$ and $\rho_{W}(S \backslash W)=1 ; \rho_{0}$ will denote the trivial representation. Observe that not all such $W$ define a homomorphism, the relations of the presentation of $\Gamma$ impose restrictions on that. Notice also that, whenever we know a complete chain complex, we write it down explicitly.

4.1. Cyclic groups, $C(t)=\left(x: x^{t}=1\right)$. Allow representation:

$$
\begin{aligned}
& \operatorname{Hom}(C(2 n+1), \mathbb{Z} / 2)=\left\{\rho_{0}\right\}, \\
& \operatorname{Hom}(C(2 n), \mathbb{Z} / 2)=\left\{\rho_{0}, \rho_{x}\right\}
\end{aligned}
$$

In this case we have a full periodic resolution, see [3] or [2], that gives the chain complex

$$
\begin{aligned}
0 \longrightarrow \mathbb{Z} \Gamma[d] \underset{d_{3}}{\longrightarrow} & \mathbb{Z} \Gamma[c] \underset{d_{2}}{\longrightarrow} \mathbb{Z} \Gamma[b] \underset{d_{1}}{\longrightarrow} \mathbb{Z} \Gamma[a] \longrightarrow 0 \\
& d_{1}(b)=(x-1) a \\
d_{2}(c)= & \left(1+x+x^{2}+\cdots+x^{t-1}\right) b \\
& d_{3}(d)=(x-1) c .
\end{aligned}
$$

The homology groups in the above representations are

\begin{tabular}{c|cccc}
\hline & \multicolumn{4}{|c}{ Twisted homology } \\
\hline & $H_{0}$ & $H_{1}$ & $H_{2}$ & $H_{3}$ \\
\hline$\rho_{0}$ & $\mathbb{Z}$ & $\mathbb{Z} / t$ & 0 & $\mathbb{Z}$ \\
$\rho_{x}$ & $\mathbb{Z} / 2$ & 0 & $\mathbb{Z} / 2$ & 0 \\
\hline
\end{tabular}


while the cohomology with global $\mathbb{Z} / 2$ coefficients is

\begin{tabular}{cccc}
\hline \multicolumn{4}{c}{$\mathbb{Z} / 2$-cohomology } \\
\hline$H^{0}$ & $H^{1}$ & $H^{2}$ & $H^{3}$ \\
\hline $\mathbb{Z} / 2$ & $\mathbb{Z} /(2, t)$ & $\mathbb{Z} /(2, t)$ & $\mathbb{Z} / 2$ \\
\hline
\end{tabular}

where $(n, m)$ denotes the gcd of $n$ and $m$.

4.2. Generalized quaternionic groups, $Q(4 t)=\left(x, y: x^{t}=y^{2}, x y x=y\right)$. Allow representation:

$$
\begin{gathered}
\operatorname{Hom}(Q(8 n), \mathbb{Z} / 2)=\left\{\rho_{0}, \rho_{x}, \rho_{y}, \rho_{x, y}\right\}, \\
\operatorname{Hom}(Q(8 n+4), \mathbb{Z} / 2)=\left\{\rho_{0}, \rho_{y}\right\}, \\
0 \longrightarrow \mathbb{Z} \Gamma[d] \underset{d_{3}}{\longrightarrow} \mathbb{Z} \Gamma\left[c_{1}, c_{2}\right] \underset{d_{2}}{\longrightarrow} \mathbb{Z} \Gamma\left[b_{1}, b_{2}\right] \underset{d_{1}}{\longrightarrow} \mathbb{Z} \Gamma[a] \longrightarrow 0, \\
d_{1}\left(b_{1}\right)=(x-1) a, \quad d_{1}\left(b_{2}\right)=(y-1) a, \\
d_{2}\left(c_{1}\right)=\left(1+x+x^{2}+\cdots+x^{t-1}\right) b_{1}+(-y-1) b_{2}, \\
d_{2}\left(c_{2}\right)=(x y+1) b_{1}+(x-1) b_{2}, \\
d_{3}(d)=(x-1) c_{1}+(-x y+1) c_{2} .
\end{gathered}
$$

The homology groups in the above representations are

\begin{tabular}{c|cccc}
\hline & \multicolumn{4}{|c}{ Twisted homology } \\
\hline & $H_{0}$ & $H_{1}$ & $H_{2}$ & $H_{3}$ \\
\hline$\rho_{0}$ & $\mathbb{Z}$ & $\mathbb{Z} / 2 \oplus \mathbb{Z} /(2, t)$ & 0 & $\mathbb{Z}$ \\
$\rho_{x}$ & $\mathbb{Z} / 2$ & $\mathbb{Z} / 2$ & $\mathbb{Z} / 2$ & 0 \\
$\rho_{y}$ & $\mathbb{Z} / 2$ & $\mathbb{Z} / t$ & $\mathbb{Z} / 2$ & 0 \\
$\rho_{x, y}$ & $\mathbb{Z} / 2$ & $\mathbb{Z} / 2$ & $\mathbb{Z} / 2$ & 0 \\
\hline
\end{tabular}

while the cohomology with global $\mathbb{Z} / 2$ coefficients is

$\mathbb{Z} / 2$-cohomology

\begin{tabular}{cccc}
\hline$H^{0}$ & $H^{1}$ & $H^{2}$ & $H^{3}$ \\
\hline $\mathbb{Z} / 2$ & $\mathbb{Z} / 2 \oplus \mathbb{Z} /(2, t)$ & $\mathbb{Z} / 2 \oplus \mathbb{Z} / 2$ & $\mathbb{Z} / 2$ \\
\hline
\end{tabular}


6 Vector bundles over three-dimensional spherical space forms

4.3. The binary tetrahedral group, $T^{*}(24)=\left(x, y: y x y=x^{2}, x y x=y^{2}\right)$.

$$
\begin{gathered}
\operatorname{Hom}\left(T^{*}(24), \mathbb{Z} / 2\right)=\left\{\rho_{0}\right\}, \\
\cdots \longrightarrow \mathbb{Z} \Gamma[d] \underset{d_{3}}{\longrightarrow} \mathbb{Z} \Gamma\left[c_{1}, c_{2}\right] \underset{d_{2}}{\longrightarrow} \mathbb{Z} \Gamma\left[b_{1}, b_{2}\right] \underset{d_{1}}{\longrightarrow} \mathbb{Z} \Gamma[a] \longrightarrow 0, \\
d_{1}\left(b_{1}\right)=(x-1) a, \quad d_{1}\left(b_{2}\right)=(y-1) a, \\
d_{2}\left(c_{1}\right)=(y-x-1) b_{1}+(1+y x) b_{2}, \\
d_{2}\left(c_{2}\right)=(1+x y) b_{1}+(x-y-1) b_{2}, \\
d_{3}(d)=(x-1) c_{1}+(y-1) c_{2}
\end{gathered}
$$

(the resolution has been communicated by Svengrowski and Tomoda [9]),

\begin{tabular}{l|cccc}
\hline & \multicolumn{4}{|c}{ Twisted homology } \\
\hline & $H_{0}$ & $H_{1}$ & $H_{2}$ & $H_{3}$ \\
\hline$\rho_{0}$ & $\mathbb{Z}$ & $\mathbb{Z}_{3}$ & 0 & $\mathbb{Z}$ \\
\hline
\end{tabular}

\begin{tabular}{cccc}
\hline \multicolumn{4}{c}{$\mathbb{Z} / 2$-cohomology } \\
\hline$H^{0}$ & $H^{1}$ & $H^{2}$ & $H^{3}$ \\
\hline $\mathbb{Z} / 2$ & 0 & 0 & $\mathbb{Z} / 2$ \\
\hline
\end{tabular}

4.4. The binary octahedral group, $O^{*}(48)=\left(x, y: x y x=y x y, x y^{2} x=y^{2}\right)$.

$$
\begin{gathered}
\operatorname{Hom}\left(O^{*}(48), \mathbb{Z} / 2\right)=\left\{\rho_{0}, \rho_{x, y}\right\}, \\
\cdots \longrightarrow \mathbb{Z} \Gamma[d] \underset{d_{3}}{\longrightarrow} \mathbb{Z} \Gamma\left[c_{1}, c_{2}\right] \underset{d_{2}}{\longrightarrow} \mathbb{Z} \Gamma\left[b_{1}, b_{2}\right] \underset{d_{1}}{\longrightarrow} \mathbb{Z} \Gamma[a] \longrightarrow 0, \\
d_{1}\left(b_{1}\right)=(x-1) a, \quad d_{1}\left(b_{2}\right)=(y-1) a, \\
d_{2}\left(c_{1}\right)=(1-y+x y) b_{1}+(x-1-y x) b_{2}, \\
d_{2}\left(c_{2}\right)=\left(1+x y^{2}\right) b_{1}+(x-1-y+x y) b_{2}, \\
d_{3}(d)=(1-x y) c_{1}+(y-1) c_{2}
\end{gathered}
$$


Esdras Teixeira Costa et al. 7

(the resolution has been communicated by Svengrowski and Tomoda [9]),

\begin{tabular}{|c|c|c|c|c|}
\hline & \multicolumn{4}{|c|}{ Twisted homology } \\
\hline & $H_{0}$ & $H_{1}$ & $\mathrm{H}_{2}$ & $\mathrm{H}_{3}$ \\
\hline$\rho_{0}$ & $\mathbb{Z}$ & $\mathbb{Z} / 2$ & 0 & $\mathbb{Z}$ \\
\hline$\rho_{x, y}$ & $\mathbb{Z} / 2$ & $\mathbb{Z} / 3$ & $\mathbb{Z} / 2$ & 0 \\
\hline \multicolumn{5}{|c|}{$\mathbb{Z} / 2$-cohomology } \\
\hline$H^{0}$ & $H^{1}$ & & & $H^{3}$ \\
\hline $\mathbb{Z} / 2$ & $\mathbb{Z} / 2$ & & & $\mathbb{Z} / 2$ \\
\hline
\end{tabular}

4.5. The binary icosahedral group, $I^{*}(120)=\left(x, y: x y^{2} x=y x y, y x^{2} y=x y x\right)$.

$$
\begin{gathered}
\operatorname{Hom}\left(I^{*}(120), \mathbb{Z} / 2\right)=\left\{\rho_{0}\right\}, \\
\cdots \longrightarrow \mathbb{Z} \Gamma[d] \underset{d_{3}}{\longrightarrow} \mathbb{Z} \Gamma\left[c_{1}, c_{2}\right] \underset{d_{2}}{\longrightarrow} \mathbb{Z} \Gamma\left[b_{1}, b_{2}\right] \underset{d_{1}}{\longrightarrow} \mathbb{Z} \Gamma[a] \longrightarrow 0, \\
d_{1}\left(b_{1}\right)=(x-1) a, \quad d_{1}\left(b_{2}\right)=(y-1) a, \\
d_{2}\left(c_{1}\right)=\left(1-y+x y^{2}\right) b_{1}+(-1+x+x y-y x) b_{2}, \\
d_{2}\left(c_{2}\right)=(-1+y+y x-x y) b_{1}+\left(1-x+y x^{2}\right) b_{2}, \\
d_{3}(d)=(1-y x) c_{1}+(1-x y) c_{2}
\end{gathered}
$$

(the resolution has been communicated by Svengrowski and Tomoda [9]),

\begin{tabular}{c|cccc}
\hline & \multicolumn{4}{|c}{ Twisted homology } \\
\hline & $H_{0}$ & $H_{1}$ & $H_{2}$ & $H_{3}$ \\
\hline$\rho_{0}$ & $\mathbb{Z}$ & 0 & 0 & $\mathbb{Z}$ \\
\hline
\end{tabular}

$\mathbb{Z} / 2$-cohomology

\begin{tabular}{cccc}
\hline$H^{0}$ & $H^{1}$ & $H^{2}$ & $H^{3}$ \\
\hline $\mathbb{Z} / 2$ & 0 & 0 & $\mathbb{Z} / 2$
\end{tabular}


8 Vector bundles over three-dimensional spherical space forms

4.6. $C(2 n+1) \rtimes C\left(2^{k}\right)=\left(x, y: x^{2^{k}}=y^{2 n+1}=1, x y x^{-1}=y^{-1}\right), k \geq 2, n \geq 1$.

$$
\begin{gathered}
\operatorname{Hom}\left(C(2 n+1) \rtimes C\left(2^{k}\right), \mathbb{Z} / 2\right)=\left\{\rho_{0}, \rho_{x}\right\}, \\
\cdots \underset{d_{3}}{\longrightarrow} \mathbb{Z} \Gamma\left[c_{1}, c_{2}, c_{3}\right] \underset{d_{2}}{\longrightarrow} \mathbb{Z} \Gamma\left[b_{1}, b_{2}\right] \underset{d_{1}}{\longrightarrow} \mathbb{Z} \Gamma[a] \longrightarrow 0, \\
d_{1}\left(b_{1}\right)=(x-1) a, \quad d_{1}\left(b_{2}\right)=(y-1) a, \\
d_{2}\left(c_{1}\right)=\left(1+x+\cdots+x^{2^{k}-1}\right) b_{1}, \quad d_{2}\left(c_{2}\right)=\left(1+y+\cdots+y^{2 n}\right) b_{2}, \\
d_{2}\left(c_{3}\right)=\left(1-y^{-1}\right) b_{1}+\left(x+y^{-1}\right) b_{2},
\end{gathered}
$$

\begin{tabular}{c|cccc}
\hline & \multicolumn{4}{|c}{ Twisted homology } \\
\hline & $H_{0}$ & $H_{1}$ & $H_{2}$ & $H_{3}$ \\
\hline$\rho_{0}$ & $\mathbb{Z}$ & $\mathbb{Z} / 2^{k}$ & 0 & $\mathbb{Z}$ \\
$\rho_{x}$ & $\mathbb{Z} / 2$ & $\mathbb{Z} /(2 n+1)$ & $\mathbb{Z} / 2$ & 0 \\
\hline
\end{tabular}

\begin{tabular}{llcl}
\hline \multicolumn{4}{c}{$\mathbb{Z} / 2$-cohomology } \\
\hline$H^{0}$ & $H^{1}$ & $H^{2}$ & $H^{3}$ \\
\hline $\mathbb{Z} / 2$ & $\mathbb{Z} / 2$ & $\mathbb{Z} / 2 \oplus \mathbb{Z} / 2$ & $\mathbb{Z} / 2$ \\
\hline
\end{tabular}

4.7. $Q(8) \rtimes C\left(3^{k}\right)=\left(x, y, z: x^{2}=(x y)^{2}=y^{2}, z^{3^{k}}=1, z x z^{-1}=y, z y z^{-1}=x y\right), k \geq 1$.

$$
\begin{gathered}
\operatorname{Hom}\left(Q(8) \rtimes C\left(3^{k}\right), \mathbb{Z} / 2\right)=\left\{\rho_{0}\right\}, \\
\cdots \longrightarrow \mathbb{Z} \Gamma\left[c_{1}, c_{2}, c_{3}, c_{4}, c_{5}\right] \underset{d_{2}}{\longrightarrow} \mathbb{Z} \Gamma\left[b_{1}, b_{2}, b_{3}\right] \underset{d_{1}}{\longrightarrow} \mathbb{Z} \Gamma[a] \longrightarrow 0, \\
d_{1}\left(b_{1}\right)=(x-1) a, \quad d_{1}\left(b_{2}\right)=(y-1) a, \quad d_{1}\left(b_{3}\right)=(z-1) a, \\
d_{2}\left(c_{1}\right)=\left(1+x y-x y x y x^{-1}-x y x y x^{-2}\right) b_{1}+(x+x y x) b_{2}, \\
d_{2}\left(c_{2}\right)=(1+x y) b_{1}+\left(x-x y x y^{-1}\right) b_{2}, \quad d_{2}\left(c_{3}\right)=\left(1+z+\cdots+z^{3^{k}-1}\right) b_{3}, \\
d_{2}\left(c_{4}\right)=(z) b_{1}+\left(-z x z^{-1} y^{-1}\right) b_{2}+\left(1-z x z^{-1}\right) b_{3}, \\
d_{2}\left(c_{5}\right)=\left(-z y z^{-1} y^{-1} x^{-1}\right) b_{1}+\left(z-z y z^{-1} y^{-1}\right) b_{2}+\left(1-z y z^{-1}\right) b_{3},
\end{gathered}
$$

\begin{tabular}{c|cccc}
\hline & \multicolumn{4}{|c}{ Twisted homology } \\
\hline & $H_{0}$ & $H_{1}$ & $H_{2}$ & $H_{3}$ \\
\hline$\rho_{0}$ & $\mathbb{Z}$ & $\mathbb{Z} / 3^{k}$ & 0 & $\mathbb{Z}$ \\
\hline
\end{tabular}

$\mathbb{Z} / 2$-cohomology

\begin{tabular}{cccc}
\hline$H^{0}$ & $H^{1}$ & $H^{2}$ & $H^{3}$ \\
\hline $\mathbb{Z} / 2$ & 0 & 0 & $\mathbb{Z} / 2$ \\
\hline
\end{tabular}


Table 5.1

\begin{tabular}{|c|c|c|c|}
\hline \multicolumn{2}{|c|}{$\Gamma$} & $\operatorname{Vect}_{1}\left(S_{\Gamma}\right)$ & $\operatorname{Vect}_{2}\left(S_{\Gamma}\right)$ \\
\hline$C(t)$ & $\begin{array}{c}t=2 n \\
t=2 n+1\end{array}$ & $\begin{array}{c}1 \\
1+\alpha_{x} \\
1\end{array}$ & $\begin{array}{c}1+m \beta, 0 \leq m<t \\
1+\alpha_{x} \\
1+m \beta, 0 \leq m<t\end{array}$ \\
\hline$Q(4 t)$ & $t=2 n$ & $\begin{array}{c}1 \\
1+\alpha_{x} \\
1+\alpha_{y} \\
1+\alpha_{x}+\alpha_{y} \\
1 \\
1+\alpha_{y}\end{array}$ & $\begin{array}{c}1,1+\beta_{1}, 1+\beta_{2}, 1+\beta_{1}+\beta_{2} \\
1+\alpha_{x}, 1+\alpha_{x}+\beta \\
1+\alpha_{y}+m \beta, 0 \leq m<t \\
1+\alpha_{x}+\alpha_{y}, 1+\alpha_{x}+\alpha_{y}+\beta \\
1,1+\beta_{1}, 1+\beta_{2}, 1+\beta_{1}+\beta_{2} \\
1+\alpha_{y}+m \beta, 0 \leq m<t\end{array}$ \\
\hline$T^{*}(24)$ & & 1 & $1+m \beta, 0 \leq m<3$ \\
\hline$O^{*}(48)$ & & $\begin{array}{c}1 \\
1+\alpha_{x}+\alpha_{y}\end{array}$ & $\begin{array}{c}1,1+\beta \\
1+\alpha_{x}+\alpha_{y}+m \beta, 0 \leq m<3\end{array}$ \\
\hline$I^{*}(120)$ & & 1 & 1 \\
\hline$C(2 n+1) \rtimes C\left(2^{k}\right)$ & & $\begin{array}{c}1 \\
1+\alpha_{x}\end{array}$ & $\begin{array}{c}1+m \beta, 0 \leq m<2^{k} \\
1+\alpha_{x}+m \beta, 0 \leq m<2 n+1\end{array}$ \\
\hline$Q(8) \rtimes C\left(3^{k}\right)$ & & 1 & $1+m \beta, 0 \leq m<3^{k}$ \\
\hline
\end{tabular}

\section{Vector bundles over 3-dimensional spherical space forms}

In this section we give a complete enumeration of the real vector bundles of ranks 1,2, and 3 over the 3 -dimensional spherical space forms. The enumeration is given in Tables 5.1 and 5.2, where we use the following notation. In Table 5.1, for each group $\Gamma$, we list in the first column the line bundles that are counted by their Stiefel-Whitney class; thus, 1 denotes the trivial bundle. Here, the $\alpha_{s}$ are fixed generators of $H^{1}\left(S_{\Gamma} ; \mathbb{Z} / 2\right)$-note that we can identify this set with $\operatorname{Hom}(\Gamma, \mathbb{Z} / 2)$. In the second column are listed, for each line bundle with first SW class $\alpha_{s}$, the 2 bundles with the same first SW class. These 2 bundles are counted by expressions like $1+\alpha_{s}+y$, where $y$ is the obstruction class in $H^{2}\left(S_{\Gamma} ; \mathbb{Z}_{\rho_{s}}\right)$. 
Vector bundles over three-dimensional spherical space forms

Table 5.2

\begin{tabular}{|c|c|c|c|}
\hline \multicolumn{2}{|l|}{$\Gamma$} & $\operatorname{Vect}_{1}\left(S_{\Gamma}\right)$ & $\operatorname{Vect}_{3}\left(S_{\Gamma}\right)$ \\
\hline$C(t)$ & $\begin{array}{c}t=2 n \\
t=2 n+1\end{array}$ & $\begin{array}{c}1 \\
1+\alpha_{x} \\
1\end{array}$ & $\begin{array}{c}1,1+\beta \\
1+\alpha_{x}, 1+\alpha_{x}+\beta \\
1\end{array}$ \\
\hline$Q(4 t)$ & $t=2 n$ & $\begin{array}{c}1 \\
1+\alpha_{x} \\
1+\alpha_{y} \\
1+\alpha_{x}+\alpha_{y} \\
1 \\
1+\alpha_{y}\end{array}$ & $\begin{array}{c}1,1+\beta_{1}, 1+\beta_{2}, 1+\beta_{1}+\beta_{2} \\
1,1+\alpha_{x}, 1+\alpha_{x}+\beta_{1}, 1+\alpha_{x}+\beta_{2}, 1+\alpha_{x}+\beta_{1}+\beta_{2} \\
1+\alpha_{y}, 1+\alpha_{y}+\beta_{1}, 1+\alpha_{y}+\beta_{2}, 1+\alpha_{y}+\beta_{1}+\beta_{2} \\
1+\alpha_{x}+\alpha_{y}, 1+\alpha_{x}+\alpha_{y}+\beta_{1}, \\
1+\alpha_{x}+\alpha_{y}+\beta_{2}, 1+\alpha_{x}+\alpha_{y}+\beta_{1}+\beta_{2} \\
1,1+\beta_{1}, 1+\beta_{2}, 1+\beta_{1}+\beta_{2} \\
1+\alpha_{y}, 1+\alpha_{y}+\beta_{1}, 1+\alpha_{y}+\beta_{2}, 1+\alpha_{y}+\beta_{1}+\beta_{2}\end{array}$ \\
\hline$T^{*}(24)$ & & 1 & 1 \\
\hline$O^{*}(48)$ & & $\begin{array}{c}1 \\
1+\alpha_{x}+\alpha_{y}\end{array}$ & $\begin{array}{c}1,1+\beta \\
1+\alpha_{x}+\alpha_{y}, 1+\alpha_{x}+\alpha_{y}+\beta\end{array}$ \\
\hline$I^{*}(120)$ & & 1 & 1 \\
\hline$C(2 n+1) \rtimes C\left(2^{k}\right)$ & & $\begin{array}{c}1 \\
1+\alpha_{x}\end{array}$ & $\begin{array}{c}1,1+\beta_{1}, 1+\beta_{2}, 1+\beta_{1}+\beta_{2} \\
1+\alpha_{x}, 1+\alpha_{x}+\beta_{1}, 1+\alpha_{x}+\beta_{2}, 1+\alpha_{x}+\beta_{1}+\beta_{2}\end{array}$ \\
\hline$Q(8) \rtimes C\left(3^{k}\right)$ & & 1 & 1 \\
\hline
\end{tabular}

Here, the $\beta_{i}$ are fixed generators of $H^{2}\left(S_{\Gamma} ; \mathbb{Z}_{\rho_{s}}\right)$. In Table 5.2 appear the real vector bundles of rank 3 with the same notation (but the $\beta_{i}$ are generators of $H^{2}\left(S_{\Gamma} ; \mathbb{Z} / 2\right)$ ).

\section{References}

[1] K. S. Brown, Cohomology of Groups, Graduate Texts in Mathematics, vol. 87, Springer, New York, 1982.

[2] H. Cartan and S. Eilenberg, Homological Algebra, Princeton University Press, New Jersey, 1956.

[3] K. W. Gruenberg, Resolutions by relations, Journal of the London Mathematical Society 35 (1960), 481-494.

[4] I. Hambleton and R. Lee, A four-dimensional approach to the spherical space form problem, unpublished. 
[5] H. Hopf, Zum Clifford-Kleinschen Raumproblem, Mathematische Annalen 95 (1925), no. 1, 313 339.

[6] L. L. Larmore, Twisted cohomology and enumeration of vector bundles, Pacific Journal of Mathematics 30 (1969), 437-457.

[7] A. Minatta, R. Piccinini, and M. Spreafico, A note about the isotropy groups of 2-plane bundles over closed surfaces, Collectanea Mathematica 54 (2003), no. 3, 283-291.

[8] H. Seifert and W. Threlfall, A Textbook of Topology, Pure and Applied Mathematics, vol. 89, Academic Press, New York, 1980.

[9] P. Svengrowski and S. Tomoda, private communication.

[10] J. A. Wolf, Spaces of Constant Curvature, McGraw-Hill, New York, 1967.

Esdras Teixeira Costa: ICMC-USP, 13560-970 São Carlos, Brazil

E-mail address: sdrs@icmc.usp.br

Oziride Manzoli Neto: ICMC-USP, 13560-970 São Carlos, Brazil

E-mail address: ozimneto@icmc.usp.br

Mauro Spreafico: ICMC-USP, 13560-970 São Carlos, Brazil

E-mail address: mauros@icmc.usp.br 


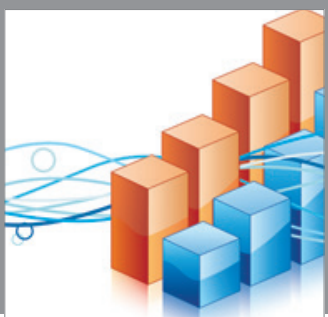

Advances in

Operations Research

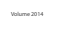

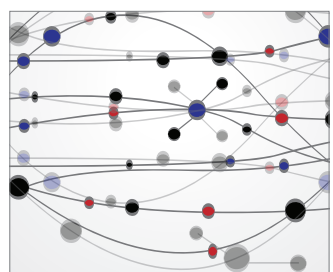

\section{The Scientific} World Journal
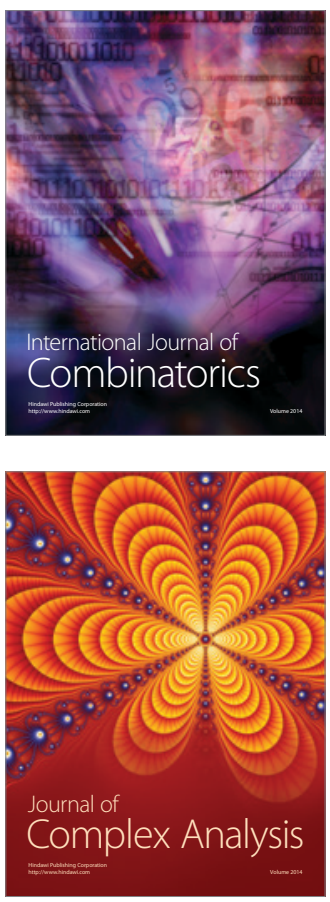

International Journal of

Mathematics and

Mathematical

Sciences
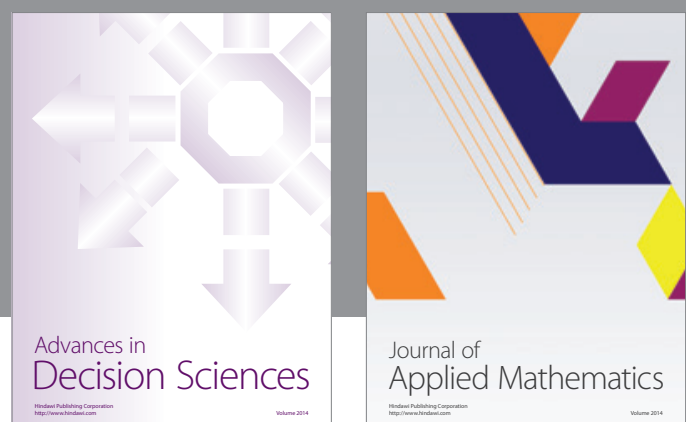

Journal of

Applied Mathematics
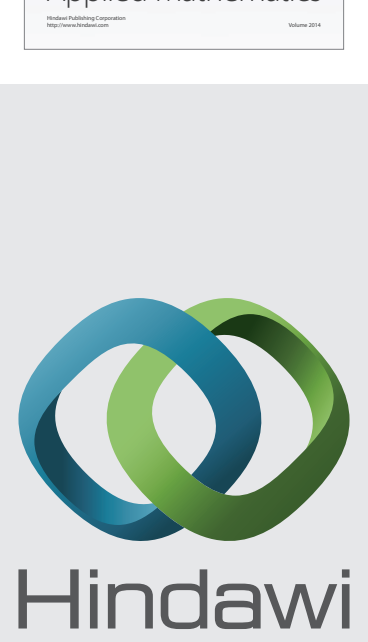

Submit your manuscripts at http://www.hindawi.com
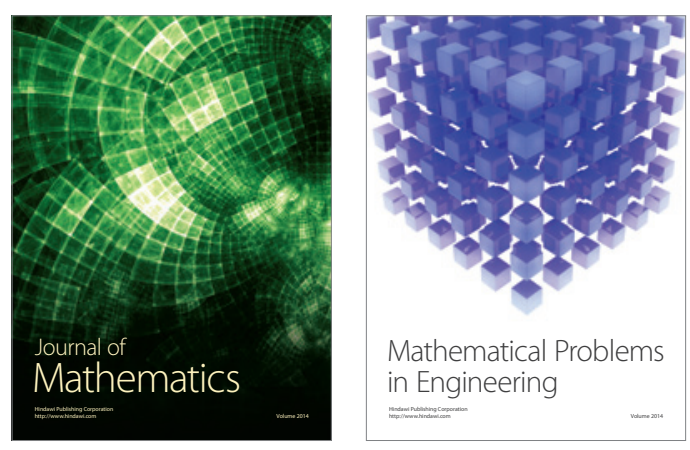

Mathematical Problems in Engineering
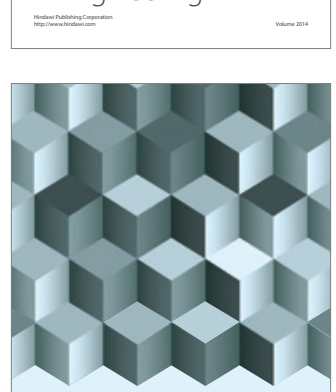

Journal of

Function Spaces
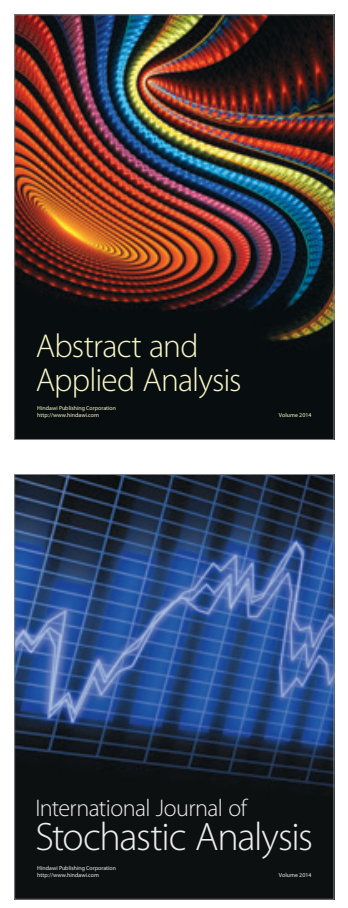

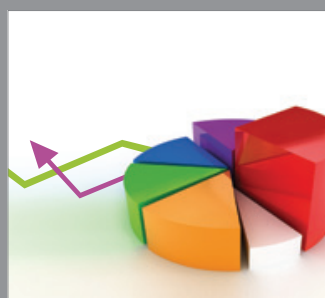

ournal of

Probability and Statistics

Promensencen
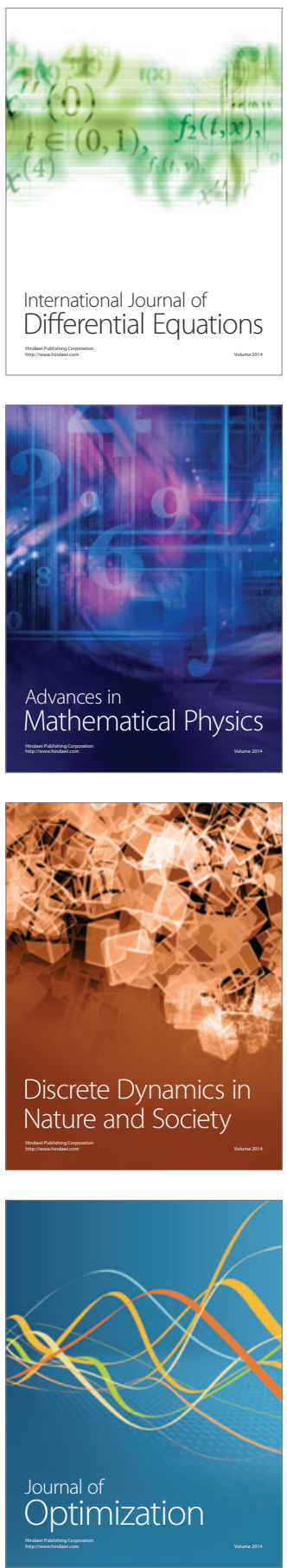